\title{
A Mixed Method Study on the Effectiveness of Using Virtual Reality to Improve Adolescent Public Speaking
}

\author{
Amber Frantz ${ }^{1}$ and Kimberly Grosenbacher" \\ ${ }^{1}$ Samuel V. Champion High School, Boerne, TX, USA \\ "Advisor
}

ABSTRACT

This study analyzes the extent to which virtual reality technology is effective in improving self-confidence in children and adolescents ages 12-18 when public speaking. Using a mixed method of both quantitative and qualitative data, subject responses were collected through a pre- and post-test survey prior to and after completing a set of three virtual reality simulations. The data demonstrated that with an increasing number of audience members present in a virtual simulation, subject confidence levels decreased, suggesting that virtual reality can be used as an effective tool in reducing public speaking anxiety. While the current study supports this claim, additional research should be conducted based on the limitations of this study, specifically to enlarge the sample size beyond 20 subjects.

\section{Introduction}

The crippling feeling of uncontrollable anxiety is rapidly increasing in prevalence in today's society, as the term "anxious" is categorized with the vast majority of social phobias. Perhaps one of the most common and relevant social phobias in relation to all ages and demographics is public speaking anxiety, as it affects approximately $70 \%$ of the population to various extents (Arnold, 2018). Public speaking anxiety (PSA) can be defined as the fear, nervousness, or anxiousness an individual may feel when faced with speaking in front of a live audience; this fear may often be accompanied by an increase in heart rate or short, rapid breathing. The science behind this common fear is rooted in the brain's fight or flight response; when faced with a fearful situation, the body triggers a "stress response [that] causes physiologic and behavioral changes that include the nervous, endocrine, and immune systems" (Chu et al., 2020). In addition, it should be noted that this form of paranoia is only "more likely to occur in people who view themselves negatively in comparison with other people" (Freeman et al., 2014). Thus, this problem of an increase in PSA prompts the need for a solution directly targeted towards its reduction or eradication.

When considering the commonality of technology in today's society, it is vital to note the disadvantageous social-emotional effects that occur as a result of heightened technological advancement. The recurring theme of "hiding behind the screen" has rapidly emerged with the aid of instant messaging programs, thus resulting in a significant decrease in self-confidence when it comes to physical confrontation (Scruton, 2010). This concept remains true when applied to the skill of public speaking, as an increased reliance on wide scale technological communication jeopardizes the ability to address a live audience. However, the solution to combating PSA ironically lies in the use of technology, more specifically, the use of virtual reality (VR). In this context, VR is often comparable to its typical use as a form of video game entertainment and can be defined as a digitally produced simulation in which the viewer is submerged into a three-dimensional world, aiding in a sense of respective realism. This false sense of reality is achieved through the use of a handheld smartphone and VR headset that is worn over the eyes. Essentially, overcoming a fear of public speaking is rooted in the repeated practice of inducing an uncomfortable situation until an individual becomes accustomed to a newfound sense of familiarity. This concept of building up a tolerance to a once-perceived intolerable situation is referred to as virtual reality exposure therapy (VRET), as participants undergo "individualized, gradual, 
controlled, immersive exposure that is easy for therapists to implement" (Boeldt et al., 2019). In order to further elaborate on this topic, it is important to note that viewers are essentially "cut-off" from the surrounding environment and are immersed in a pixelated world that is teeming with artificial, graphic life (Pan \& Hamilton, 2018). The ability for a VR system to respond and adapt to an individual's unique response is critical for advanced simulations; this ultimately results in a more personalized experience and elevates the potential this technology has in terms of serving as a form of therapy for PSA.

\section{Literature Review}

A comprehensive review of past literature serves to analyze the methods and approaches as well as results of studies concerning the variables of VR and PSA. The following three studies address the use of VR to increase self-confidence and combat PSA with the design of a virtually simulated audience.

The first study addresses the difference between a virtually simulated audience that is relatively "attentive, well-behaved, and interested" versus one that exhibits a negative connotation of body language such as " loud yawning, turning away, [and] falling asleep" (Slater et al., 1999). Dr. Mel Slater, a professor and researcher at the University of Barcelona, addresses this questionable distinction in his methodology. The quantitative method is conducted in a two-factor system in which the VR viewer first experienced an attentive audience that lacked disruption; the second factor consisted of a virtual audience that had "hostile reactions" and significant disruptions (Slater et al., 1999). Each participant repeated the two factors three times while reciting a memorized speech in order to minimize the margin of error, thus obtaining an average result between the three trials. The results from this study were accumulated and gathered through the use of a self-rating system. A low self-rating can be attributed to a "negative audience... [and] minimum perceived audience interest" and a high self-rating is accredited to two different aspects: one, a "negative audience... [and] highest perceived interest" or a "positive audience and highest co-presence" (Slater et al., 1999). It is concluded that there is an $89 \%$ variation in the self-ratings due to the fact that "when the audience is actually negative, perceived audience interest can overcome the negativity" (Slater et al., 1999). This concept is further elaborated on by Dr. Xueni Pan in the Department of Computing at the University of London, UK and Antonia F. de C. Hamilton at the Institute of Cognitive Neuroscience, UCL, UK. It is argued with the full evaluation of a technological lens, that the more responsive the software, the more likely for the production of realistic results as well as substantial improvement in measured variables unique to a specific study (Pan \& Hamilton, 2018).

Dr. Debra Boeldt at the University of Colorado, Denver expands on this theme and concept of exposure therapy by presenting the obstacles associated with this technique. The primary concern is "patient fears of exposure therapy;" the concept of voluntarily inducing a stressful and fearful simulation without an absolute guarantee of improvement is both daunting and risky (Boeldt et al., 2019). This leads into a second issue: therapist concerns. Boeldt explains that the chief concern therapists pose is purposeful distress that may result in and "increase patient drop out" (2019). However, it is explained that the benefits VR provides for future innovation outweigh the drawbacks. It is often assumed that the cost of VR is astronomical and too unrealistic to have the capability to produce quantifiable results. In actuality, Boeldt explains from an economic standpoint that the "cost of VR software and hardware continues to decline, while the quantity and quality of VR content increases" (2019). This feasibility holds great promise for the adoption of a wide range of different variables including the analysis of team building skills, reduction of paranoia related fears, and even the reduction of anxiety and improvement of self-confidence when it comes to public speaking. Boeldt emphasizes the need for both VRET and face-to-face interaction with a therapist in the field of psychology in order to produce most accurate and beneficial results. It is not suggested that VRET should "replace the need for trained therapists" (Boeldt et al., 2019).

While Slater, Boeldt, and Pan and Hamilton explain a broader context of how virtual reality can be applied to a variety of anti-anxiety related therapies, Dr. Sandra Harris of California State University analyzes the specific incorporation of VR in "reducing public speaking anxiety of university students" (2002). Harris explains through the method of a pre/post-test, VRET was used to implement a successful reduction in PSA of university-age students. In 
this study, PSA is referred to as a type of social phobia. Harris uses the following measurements to record data from the eight students that completed the VR simulation and the six in the control group: "four self-report inventories, self-report of Subjective Units of Discomfort... [and] physiological measurements of heart rate" (2002). The conclusion is derived from a distinct social perspective and is addressed in a precise and logical manner, stating that this method of virtual reality therapy was successful in reducing public speaking anxiety (Harris et al., 2002). However, a gap and limit in the research is acknowledged, as there is a significant lack of VR research on younger populations, specifically in secondary school age youth (Harris et al., 2002).

Pan and Hamilton set the parameters as to why VR is rapidly emerging as one of the leading therapeutic technologies in the field of psychology. A key concept to grasp is that VR allows for the manipulation of an "experimental control of a complex social situation" (Pan \& Hamilton, 2018). Nevertheless, the lack of research in younger age populations generates a significant research gap, as this therapeutic technology has yet to be fully explored and immersive for all ages. Dr. Philip Lindner from the Department of Psychology at Stockholm University draws attention to the severity of PSA, emphasizing that "one-third of the population reports excessive anxiety before speaking in front of an audience, and a further third reports clinically significant distress or interference with everyday life" (2020). Therefore, the use of VR to treat PSA will not only prove to be effective in raising awareness for this form of anxiety but will address the "large treatment gap for mental disorders" (Lindner et al., 2020). In terms of childhood PSA, if left untreated, there is potential for further impairments to arise in adulthood (Kahlon et al., 2019). This paper seeks to justify that there is in fact a significant prevalence of PSA amongst children ages 12-18 and that VR offers a potential form of therapy to combat such anxiety. Thus, the combination of the aforementioned factors and gaps ultimately led to the essential research question: to what extent do virtual reality simulations improve self-confidence when public speaking among children and adolescents ages 12-18 in Texas Hill Country public schools? The thought process of improving self-confidence by targeting a specific age range in a distinct and localized area would ultimately reduce PSA all together.

\section{Methods}

This study was reviewed and approved by the Institutional Review Board (IRB) in order to ensure that the research discussed in this paper was done under ethical measures due to the involvement of human subjects. The purpose of this research is to establish the relationship between using VR technology as a form of therapy to combat PSA in adolescent populations and determine its overall effectiveness in a limited time frame through immediate feedback. This research explores the relationship between VRET and public speaking, thus forming a conclusion based on the effectiveness of VR technology itself.

\section{Design}

The research design centers around the inclusion of technology. This is how the hypothesis will be answered: VRET has the capability to improve public speaking abilities. The best approach to test this hypothesis is in the form of a mixed method. Due to the numerical and characteristical feedback a mixed method provides, the explorative purpose is supported through the means of precise data collection that contains no variation in the variables tested. In addition, the results can be replicated and explored over larger audiences. As a result, an experimental analysis was conducted based off of the closed ended answers obtained from a pre/post-test survey. An experimental analysis includes the use of two related variables, VR and PSA, as well as the use of a constant in order to observe whether a demonstrated change took place. This experiment involved 20 voluntary participants ranging from ages $12-18$ in public secondary schools in the Texas Hill Country (parental consent was obtained due to the involvement of minors). An age range was achieved through the inclusion of both middle school and high school populations; the idea of not concentrating on one specific age group is so that a correlation study can be conducted regarding the varying results produced by 
different ages. As a whole, this method is unique due to the fact that it both targets adolescents and was conducted in an approximate time frame of three hours for all participants combined. Studies in past literature have focused on a larger length of time, specifically that of a "pilot study with pre, post, and 1 and 3 month follow-ups" (Kahlon et al., 2019). Thus, this creates an additional gap of a narrow time frame in addition to targeting participants of a younger age group.

The study itself consisted of four nonconsecutive days in total: one day spent filming and one day conducting the experiment (two days per each campus). VR simulations, created through the use of the RICOH THETA $360^{\circ}$ camera, were used in order to observe the potential improvement in self-confidence when it comes to public speaking ability. This set up parallels that of Dr. Snežana Stupar-Rutenfrans of University College Roosevelt in which a VR simulation was developed through a " $360^{\circ}$ smartphone application for a VR head-mounted device" (2017). Prior to the study's day of execution, a set of three panoramic images were created; two sets were produced in total due to the inclusion of both a middle and high school campus. The same students who later participated in VR simulations first took part in the production of the panoramic images. The first set of images were of an empty classroom, the second were of a halfway occupied classroom with 4-5 participants, and the third were of a completely occupied classroom with 10-12 participants. In this sense, the image of the empty classroom was observed as a constant in the study in order to demonstrate a change in participants' confidence level given an increase in audience size. The idea of filming at both campuses allowed the students to view a prospective environment that was familiar and comfortable (such as a classroom they regularly attend), rather than a universal setting that is rather foreign. Once completing the panoramic images, one-by-one they were downloaded into the RICOH THETA mobile app for iPhones. This software was ideal due to the fact that the Blackfin VR headsets used were compatible with the iPhone in which all six VR simulations were on.

\section{Measures}

Prior to experiencing the series of VR simulations, participants from both campuses completed a ten question survey that centered around their current personal opinion of their public speaking capabilities. The survey(s) were of original design and were based on closed ended answers (yes/no, ranking from 1-10, etc.) in addition to qualitative questions that centered on the selection of different emotional characteristics a participant may feel towards the act of public speaking. At the top of each survey, students were also asked to record their age; this was how an accurate age range was obtained. The survey questions were universal for all ages and served as a constant in the study. The pre-test survey questions can be located in Appendix A; the inclusion of each question aids in the replication of this research method, as one would be able to obtain results from the same scales used in this study. After the surveys were turned in, students completed a series of three trials of the VR simulations one-on-one with the researcher. The idea of conducting the trials in a controlled environment with only the researcher present reduced the amount of distractions experienced in a normal classroom. Each trial consisted of the different shots taken previously concerning the occupancy of the classroom. The trials began when the student was asked to give a one minute impromptu speech while wearing the virtual reality headset. The following prompts, listed in the order given from the empty classroom VR simulation to the full classroom, were given to all participants: If you could travel through time, where would you go? Who is someone you look up to and why? Where do you see yourself in five years? The thought process of using different prompts specific to each trial was to limit the potential of memorization or rehearsal if the same prompt were to be used for all simulations. When each participant began speaking, a timer was started to record how long they were able to speak about each prompt. This process repeated as both the simulation and question changed but remained unvaried for both middle and high school campuses. Upon completion of the three trials, the participants immediately completed a post-test survey. The survey questions oriented around their experience and focused on the immediate feedback results that occur as an effect of VRET (post-test survey questions can be found in Appendix B). 


\section{Limitations}

The main gap addressed in this study can also coincide as a limitation. Because only the immediate results from the VR simulations are observed, this research lacks the long term, potentially beneficial effects that would be denoted as well. In addition, the small study pool size leads to question the generalizability of the data collected. This limitation can also be grouped with the fact that an equal number of participants were not obtained for both the middle and high school age populations (12 middle school participants versus 8 high school participants); thus, the statistics obtained from a correlational study are more difficult to compare in terms of relevance and accuracy, as an equivalence in average population size was not achieved.

\section{Delimitations}

As a result of this mixed method approach, a new analysis and conclusion can be formed. The relationship between VR technology, VRET, and public speaking is the main focus of this experiment; in addition, the correlation study between age groups is also a vital aspect in determining the overall effectiveness of VRET for different ages. Because of an age spectrum of six years amongst varying participants, a constant in the study was vital and achieved through the invariable survey questions. In addition, an equal number of participants from each age was not achieved in order to accentuate the concept of a randomized subject pool. Thus, a mixed approach, one that targeted both emotional characteristics and numerical averages, is best suited for the variables (VR and PSA) in the research question.

\section{Results}

\section{Quantitative Results}

After conducting a preliminary analysis of all data collected, it can be clearly seen that VR does in fact lead to the improvement of confidence when public speaking. This inference is justified by the fact that there is a significant decline in confidence when participants were faced with an increased number of audience members. When participants were asked to rank their self-confidence when public speaking on a scale of 1-10 (1 being the least, 10 being the greatest) prior to viewing the VR simulations, the average of all 20 responses was 6.2 . This number can be compared to the three other self-confidence values that were assessed after all VR simulations were completed. Measured on the same scale (1-10), the average confidence level when speaking while viewing the VR simulation without an audience was 8.2. However, this value decreased with an increase of audience members. For the simulation with only 4-5 audience members, the average confidence level was 7.7, while the simulation with 10-12 audience members resulted in an average confidence level of 7.3. In addition, participants were also polled during the post-test as to whether or not they would feel more confident when speaking if given prior knowledge of the impromptu speaking prompts. The vast majority of participants responded "Yes" (70\%), while 25\% selected "Some" and only 5\% chose "No."

Several other factors were assessed during the pre-test portion of this study in order to gauge a sense of participants' attitudes regarding public speaking. When asked about preferred presentation style, $70 \%$ of all respondents chose that their preferred method of presenting was in a group setting; conversely, only $30 \%$ of all respondents indicated that they preferred presenting individually. When also asked if participants thought their public speaking capabilities would improve with practice, an overwhelming majority of 85\% selected "Yes," while 15\% of participants reported that their speaking abilities would improve "Some" with more practice. Finally, participants were questioned if their fear of public speaking interfered with group participation. Results from this question were mixed, as only 
$40 \%$ of participants responded that their PSA impacted their group participation "Some," 45\% said "No," and 15\% indicated that they did not have a fear of public speaking.

Participants were asked similar questions in the post-test survey; more specifically, these questions oriented around participants' experience with the VR simulations and included thought provoking topics that serve to stimulate the discussion of future implications. It was polled as to whether or not the repeated use of VR would improve participants' public speaking capabilities. Again, this question spoke in favor of VR, as 70\% chose "Yes" and only 30\% selected "No." It was also polled as to whether or not participants would be open to using VR again in the future to help with public speaking. This question had the highest positive percentage out of the entirety of the data with $90 \%$ of participants reporting "Yes," their speaking abilities would improve with the continual practice of VRET. Finally, participants were asked whether or not they would feel more comfortable presenting after having completed the VR simulations. Results were slightly mixed from this question, as 65\% responded "Yes," $30 \%$ selected "Some," and the remaining $5 \%$ chose "No."

\section{Qualitative Results}

When assessing the qualitative portion of the data, participants were asked in the pre-test to circle all descriptive words that coincided with their attitude regarding public speaking. A similar question was posed in the post-test regarding words that participants associated with their immediate reaction with upon completing the three simulations. Figure 1 demonstrates the number of times each word was chosen (participants were not limited to a maximum of words they could circle). It can be deduced that the two most frequent words circled were "Nervousness" and "Anxious," thus verifying the statement that the vast majority of participants associate sentiments of negative connotation with the act of public speaking. Tied for the third most frequently selected words were "Pressure" and "Tense," furthering the relative theme that public speaking is regarded poorly among a younger audience of 12-18 years old. During the posttest, qualitative results were also obtained by furthering general themes relative to participants' personal opinions. Figure 2 depicts seven additional words and the number of times they were chosen with respect to participants' feelings immediately after completing the simulations. This time, results pointed towards a trend distinctly opposite than that demonstrated by Figure 1. The two most frequently chosen words were "Accomplished" and "Satisfactory," thus deducing a theme of a relatively positive connotation, one that speaks to the effectiveness of VR itself. 


\section{Number of Times Chosen vs. Descriptive Word: Pre-Test}

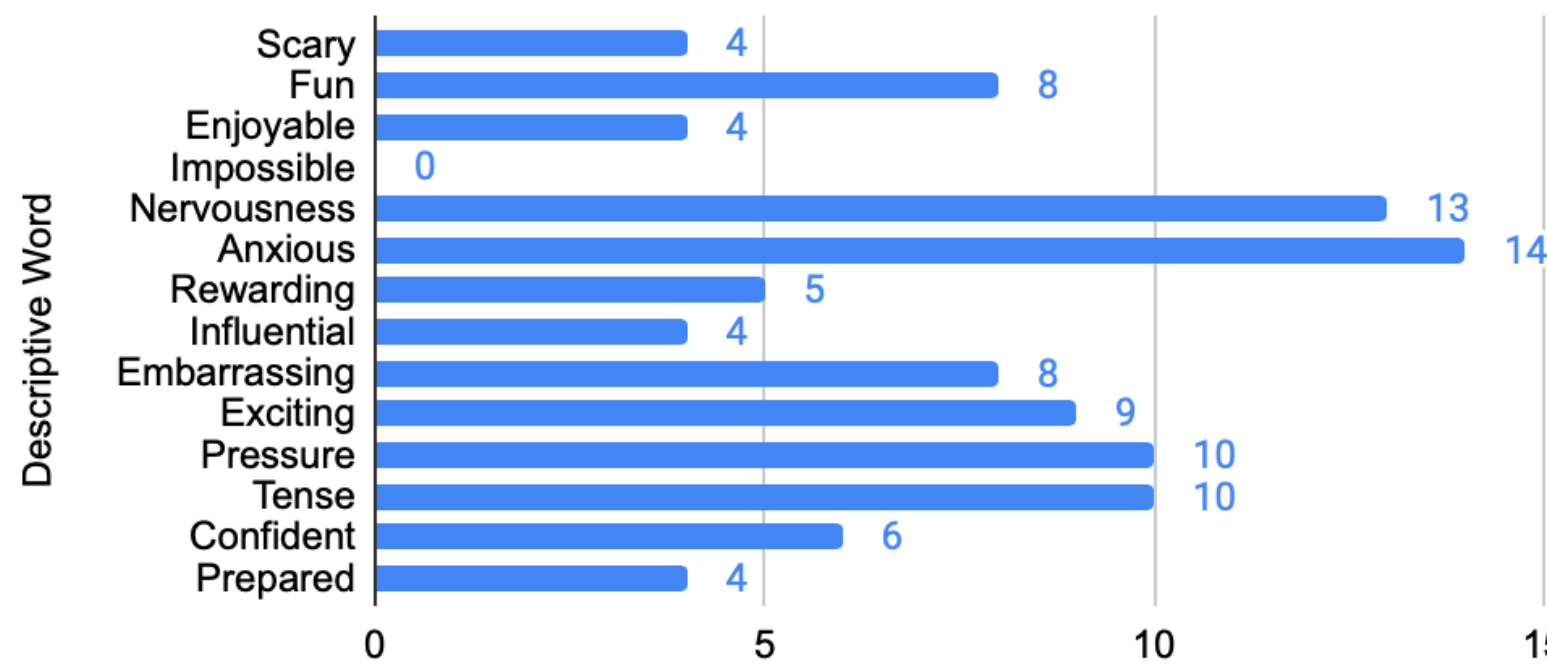

Number of Times Chosen

Figure 1. Number of Times Chosen vs. Descriptive Word: Pre-Test

\section{Number of Times Chosen vs. Descriptive Word: Post-Test}

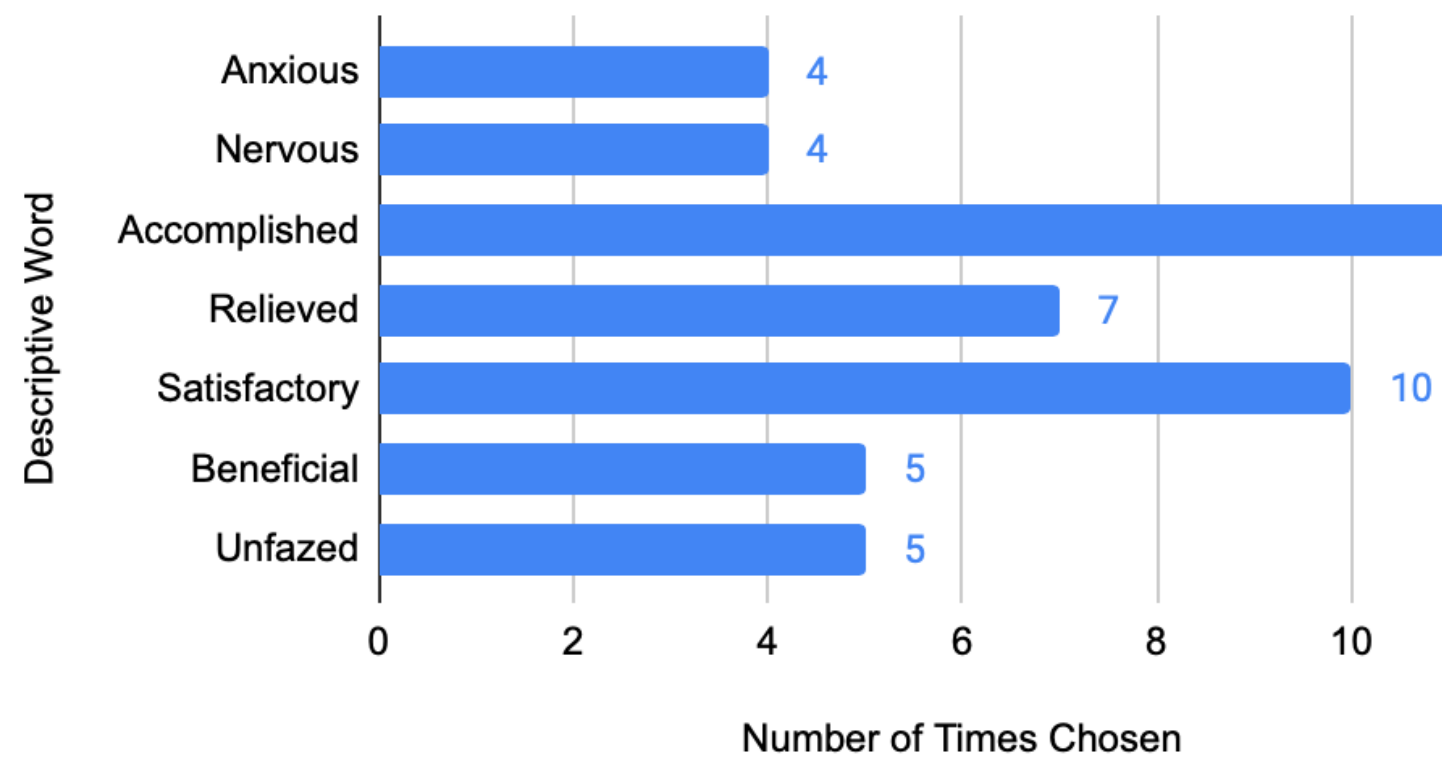

Figure 2. Number of Times Chosen vs. Descriptive Word: Post-Test 
The pre-test continued to suggest more qualitative trends in relation to the art of public speaking in general, as participants were given three statements and told to select whether they strongly disagreed, strongly agreed, disagreed, agreed, or were neutral towards them. The results from these statements were not consistently conclusive, as there was not a clear point of view that participants aligned themselves with the most in all circumstances. As observed in Figure 3, when participants were posed with the statement, "I do poorly on speeches because I am nervous," $50 \%$ of all respondents chose that they had a disposition of disagreement towards the statement, while $30 \%$ said that they felt "Neutral." Thus, the consensus that half of the respondents felt at least some level of disdain can be formed; however, this number is not conclusively significant, as it does not represent the majority of all participants. In addition, the second statement (Figure 4), "My lack of experience when it comes to delivering speeches results in a poor performance," resulted in both the "Neutral" option and overall disagreement point of view being the most frequently selected, tying at 40\% each. Conversely, the final statement (Figure 5), "I experience a fear of forgetting what I am going to say when reciting a rehearsed presentation," yielded the most promising results, as a total of 55\% of all participants demonstrated a point of view of general agreement. Therefore, a general consensus can be formed from this statement: the fear of forgetting what one is going to say in a presentation is a genuine concern of at least half of the polled population.

\section{Statement: I do poorly on speeches because I am nervous.}

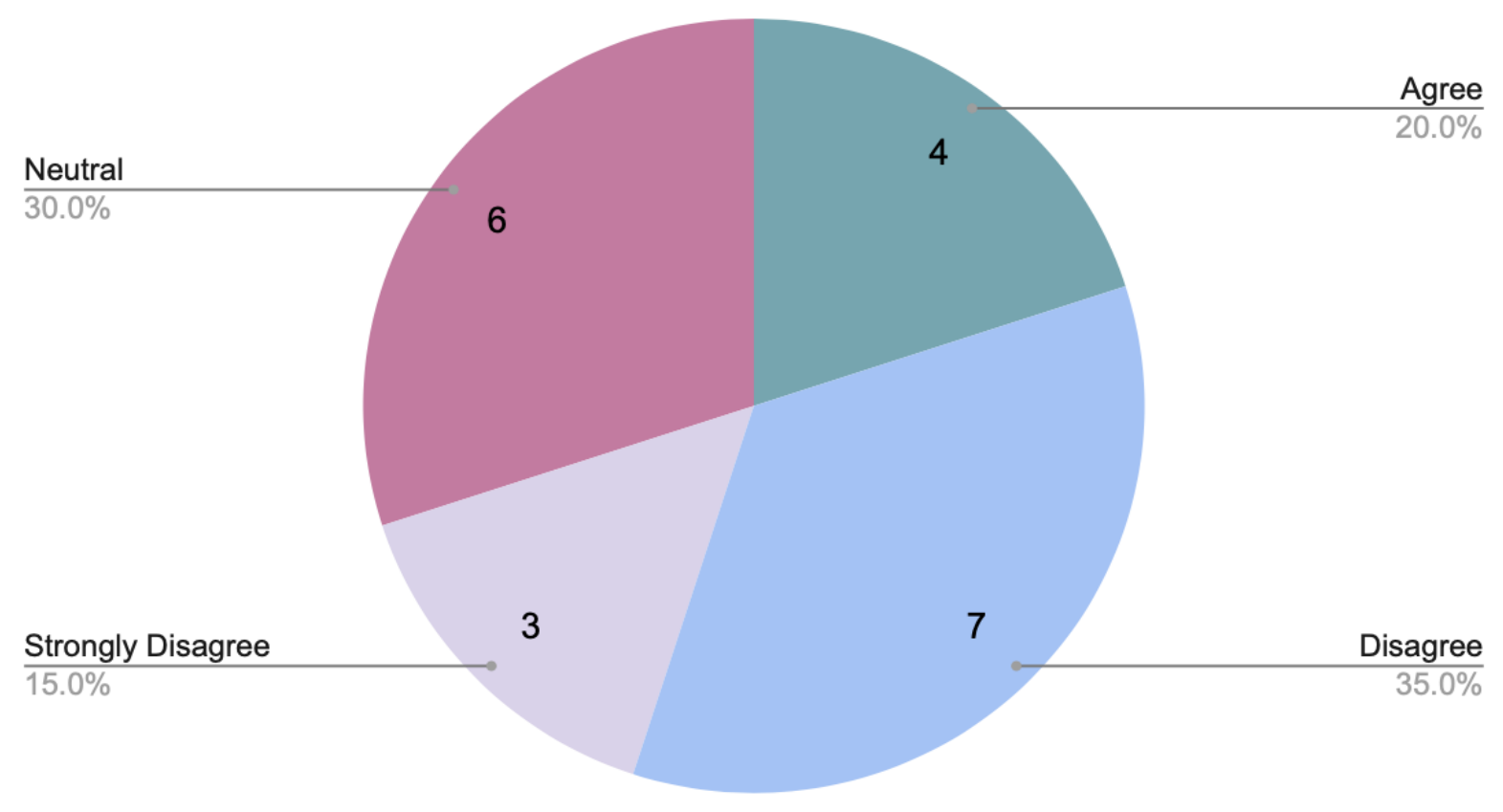

Figure 3. Statement: I do poorly on speeches because I am nervous 


\section{Statement: My lack of experience when it comes to delivering speeches results in a poor performance.}

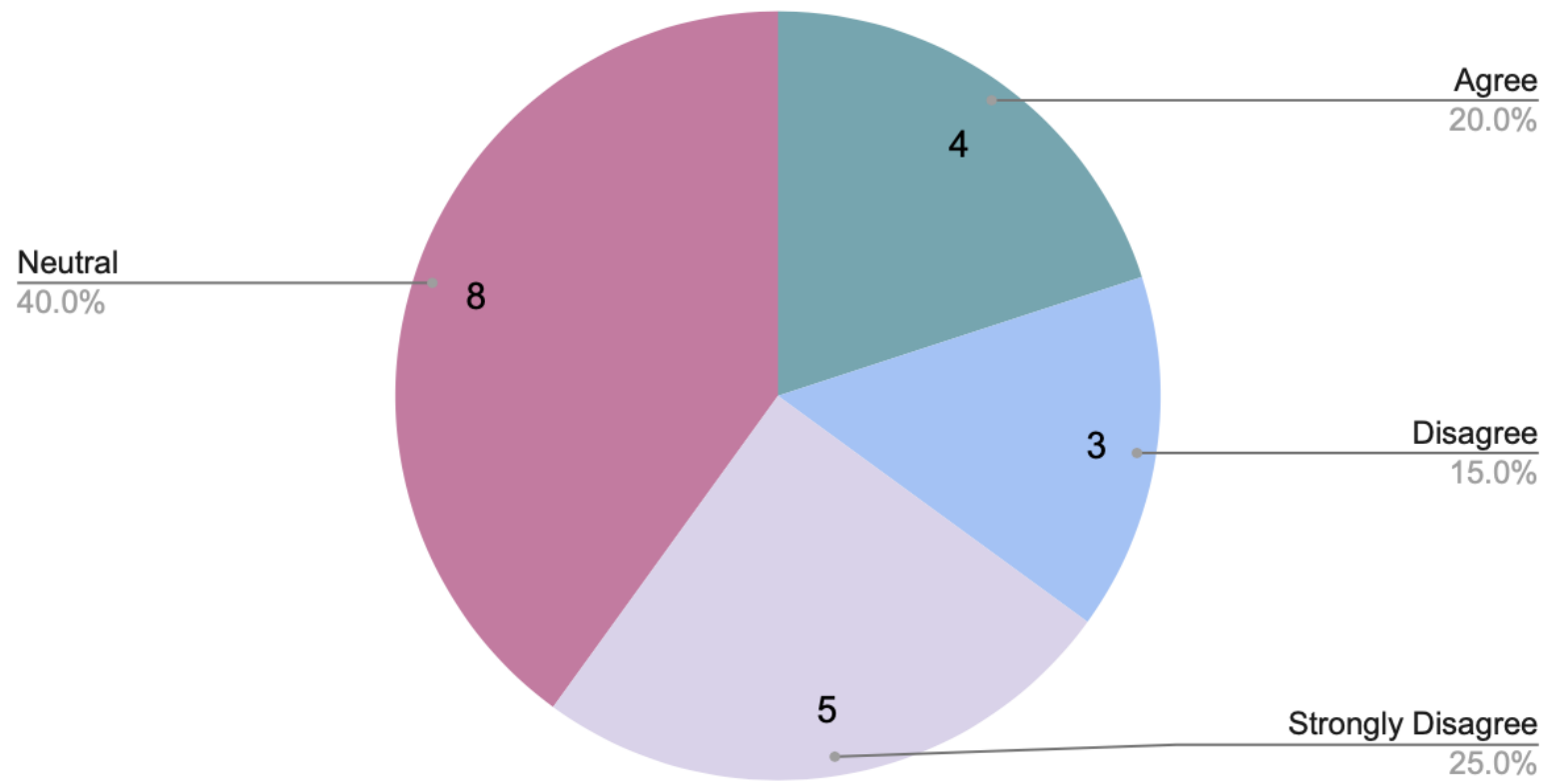

Figure 4. Statement: My lack of experience when it comes to delivering speeches results in a poor performance

\section{Statement: I experience a fear of forgetting what I am going to say when reciting a rehearsed presentation.}

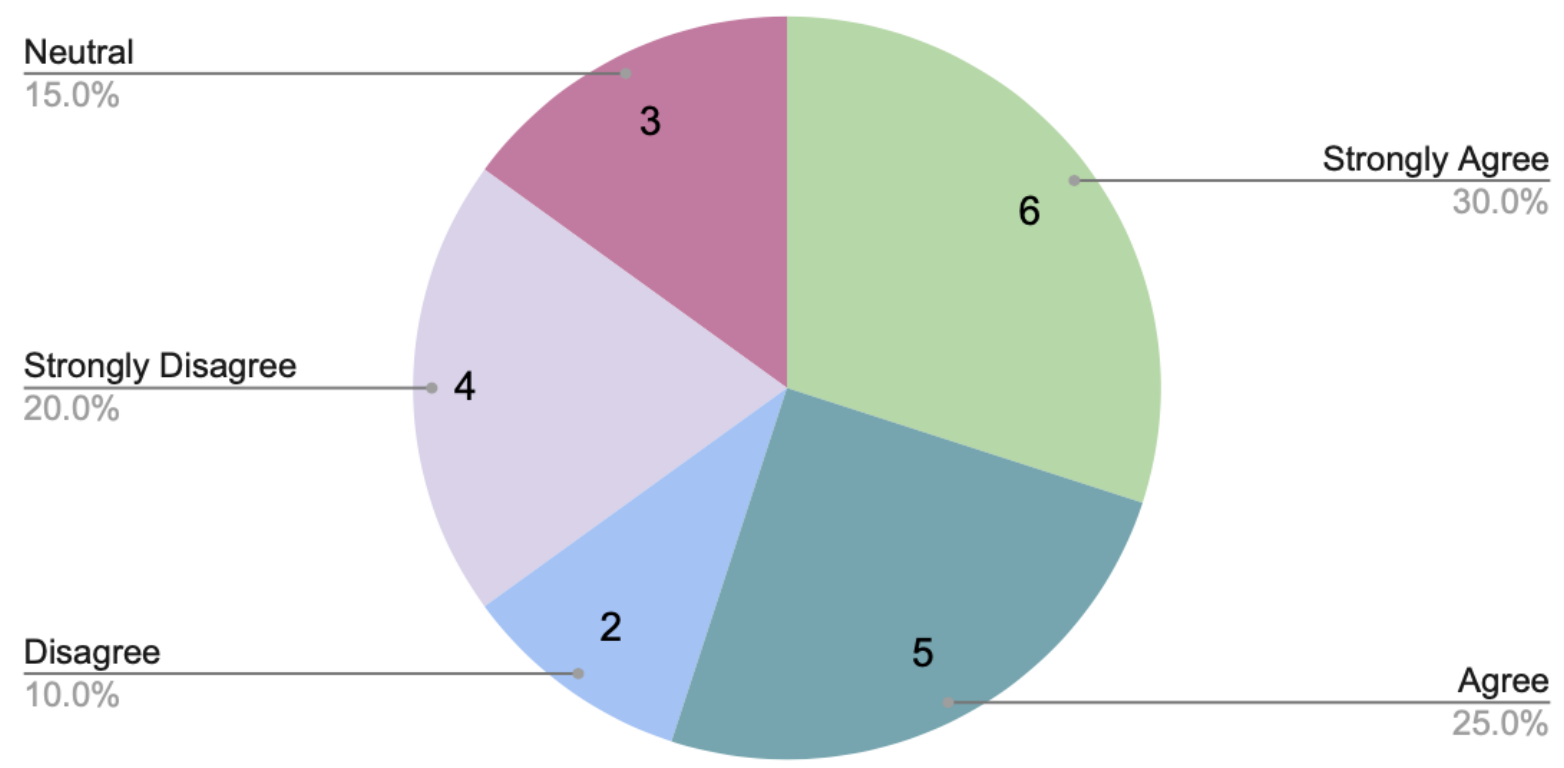

Figure 5. Statement: I experience a fear of forgetting what I am going to say when reciting a rehearsed presentation 


\section{Correlational Study}

In addition to an experimental analysis, a correlational study was conducted, assessing how results differed amongst two different age groups. The first age group centered around the middle school level of education and included ages 12-14, while the second age group reached the high school level of education and included ages 15-18. The main points of comparison were specifically how participants' level of confidence differed with respect to each age group. In the pre-test portion of the study, when asked to rank one's confidence level on a scale of 1-10, the average middle school age group of 12 participants resulted in the score of a 6; the high school age group, consisting of 8 participants, was not significantly higher, scoring in at 6.5. In the post-test, the general trend regarding confidence level decreasing with an increased number of audience members remained true for the middle school age group, as numbers polled out to be 8.7 (no audience), 7.7 (small audience), and 6.6 (large audience). However, this trend was slightly deviated in the high school age group, as average confidence levels resulted in 7.4 (no audience), 7.8 (small audience), and 7.6 (large audience). Finally, the time component of clocking how long each participant spoke for comes into play in the correlational study. The average time all participants spent talking for the combination of all three trials was 45 seconds. When taking the average time spoken for all trials in the middle school age population, the number was higher, clocking in at 49.6 seconds. Results differed drastically when taking the average time for the high school participants, as 38.1 seconds was the combined average for all three trials.

\section{Discussion}

\section{Findings}

After examining the results obtained through both the experimental analysis and correlational study, a new understanding can be concluded that VR does have the ability to improve self-confidence when public speaking amongst children and adolescents; thus, this confirms the original hypothesis and validates the research question. The overall downward trend of average confidence levels with an increase in audience members results in the statement that PSA is exacerbated when an individual is faced with a larger crowd. In addition, several other factors were assessed in order to present the final conclusion that participants have a negative view towards the act of public speaking. While the overwhelming majority of participants did not feel that their PSA interfered with group participation, a significantly large percentage indicated that they felt more confident when presenting with a group, rather than individually. Likewise, it can be inferred that with more preparation, participants would experience a lesser level of anxiety, as 95\% of all respondents reported that they would feel at least somewhat more confident when given prior knowledge of the impromptu speaking topics. The qualitative results in the pre-test portion continued to prove that participants associated the adverse feelings of nervousness and anxiety with the act of presenting. However, it can be observed in the qualitative portion of the post-test that although participants felt negatively towards the act of public speaking itself, their feelings did not impact their performance while viewing the simulations, as the vast majority reported that they felt accomplished and satisfied upon completion. In terms of the correlational study, the middle school age population paralleled the trend of confidence levels experienced by the entire group. On the other hand, the high school age group experienced an increase in average confidence levels as the simulations increased in audience members. This difference can be accounted for a potentially greater level of familiarity with the different prompts presented; participants may have felt more confident talking about one specific topic, thus resulting in an overall higher confidence level.

When considering past literature, the results of this study do parallel that of Harris' investigation, as the same conclusion is reached: VRET does have the capability to reduce PSA. However, it is difficult to compare to what extent the statistics obtained from the study discussed in this paper coincide with the numerical data Harris obtained, as two separate age groups were addressed. Nevertheless, due to the crossover of the use of a pre/post-test method as both Harris' and this study's approach, the claim can be made that VR is a beneficial form of therapy for combatting 
PSA amongst several ages, 12-22 years of age to be exact. Additionally, this study refutes the counterargument that is presented by Boeldt, in which it is proposed that VRET does more harm than good in terms of elevating the anxiety of viewers. In all actuality, the results of the study presented in this paper speak to the new understanding that participants felt that their experience was "satisfactory," as $90 \%$ reported their public speaking skills would continue to improve with the repeated practice of VRET.

\section{Implications}

The results from this study can be applied to the broader fields of neuroscience and psychology, as VRET is emerging as a common therapeutic tool to treat various phobias, much like that of PSA. Dr. Corey J. Bohil, professor for the Department of Psychology at the University of Central Florida, speaks in relation to the wide spectrum of uses and benefits of VR, noting that "[v]irtual environments can present combinations of stimuli that are not found in the natural world and researchers can execute changes in the environment that would not be possible physically" (2011). In addition to social phobias, VR has the potential to provide a greater understanding "of the theory of mind deficit that is thought to occur in Autism Spectrum Disorders" (Bohil et al., 2011). Because a virtually simulated environment is one under complete control, this form of technology can be adapted for various learning disorders including, but not limited to, autism, Asperger syndrome, and attention-deficit/hyperactivity disorder; thus, VR is advantageous for "people with [learning difficulties] in that it also offers structure, opportunities for repetition, emotional engagement and, additionally, control of the learning environment" (Vera et al., 2005). Furthermore, the increased incorporation of VR into the classroom would aid in not only the creation of social connections for students with learning disabilities, but help to challenge all students who face even minor difficulties with the primary topic discussed in this paper -PSA. Therefore, the use of VRET for children and adolescents drives neural cognitive development, ultimately leading to the hope that "VR may increasingly be viewed as an ordinary part of neuroscience research and therapy" (Bohil et al., 2011).

\section{Limitations}

As previously stated in the Method section, the main gap addressed in this study doubles as a limitation. Due to the study observing the immediate effects of VR on confidence level in relation to public speaking, there is a lack of longterm results that may potentially suggest greater benefits or detriments in relation to the overall effectiveness of VRET. This limitation is a direct consequence of the limited time frame given to complete the study. Similar to the claim proposed by Boeldt, a counterargument against the use of VR as a form of therapy altogether is presented by Smiti Kahlon, a PhD student at Haukeland University Hospital, in which it is argued that VR does more harm than good. Although this counterargument that VR does in fact "provoke stress in socially anxious youth" has been observable and is also applicable to the study presented in this paper, it is disproved by the results and findings (Kahlon et al., 2019). An additional counterargument suggested in past literature would be "that while therapists may acknowledge the many advantages of VRET, they view the technology as technically inaccessible and expensive" (Lindner et al., 2019). This counterargument, although relative to the technology of VR and broader field of psychology, was not a limitation for this paper, as the VR headset and $360^{\circ}$ camera were borrowed without monetary compensation. Arguably the largest limitation of this study would be the relative size of the subject population. Because the study pool only contained 20 participants, there is reason to believe that the statistics obtained may not be substantially relevant or representative of a larger population that includes various demographics and a greater number of participants for each age group. Finally, the last limitation addressed concerns the fact that some participants were able to speak about each impromptu topic longer than others, thus giving them more exposure to the VR simulations. 


\section{Future Studies}

This study's limitations serve as reasons for future research. Due to the study's replicability, it should be expanded to include a larger subject pool, including numerous participants for each age addressed in the spectrum. Future research should also be conducted using the parameters as discussed in this paper but open the study to a wider area of various demographics, as this study focused on an isolated region in the Texas Hill Country. Finally, one could repeat this study with the reuse of both the pre/post-test over time, allowing for the comparison of results and how they differ with the repeated practice of VRET. Thus, participants would reap the benefits of extended public speaking practice, ultimately making progress towards the gradual elimination of PSA all together.

\section{Acknowledgments}

The author would like to thank Mr. Bret Bunker of the BISD technology department for providing the VR equipment used in this study. Additional gratitude is extended to the faculty, staff, and students within BISD who participated and contributed to this study. Finally, the author would like to thank the faculty advisor on this project, Ms. Kimberly Grosenbacher.

\section{References}

Arnold, James K., "Stress to Success: Public Speaking Anxiety and its Relationship to Perceived Leadership" (2018). Education Doctoral Dissertations in Leadership. 110. https://ir.stthomas.edu/caps ed lead docdiss/110 Boeldt, D., Mcmahon, E., Mcfaul, M., \& Greenleaf, W. (2019). Using Virtual Reality Exposure Therapy to Enhance Treatment of Anxiety Disorders: Identifying Areas of Clinical Adoption and Potential Obstacles. Frontiers in Psychiatry, 10. https://doi.org/10.3389/fpsyt.2019.00773

Bohil, C. J., Alicea, B., \& Biocca, F. A. (2011). Virtual Reality in Neuroscience Research and Therapy. Nature Reviews Neuroscience, 12(12), 752-762. https://doi.org/10.1038/nrn3122

Chu, B., Marwaha, K., Sanvictores, T., \& Ayers, D. (2020). Physiology, Stress Reaction. In StatPearls. StatPearls Publishing.

Freeman, D., Evans, N., Lister, R., Antley, A., Dunn, G., \& Slater, M. (2014). Height, social comparison, and paranoia: An immersive virtual Reality experimental study. Psychiatry Research, 218(3), 348-352.

doi:10.1016/j.psychres.2013.12.014

Harris, S. R., Kemmerling, R. L., \& North, M. M. (2002). Brief Virtual Reality Therapy for Public Speaking Anxiety. CyberPsychology \& Behavior, 5(6), 543-550. http://128.192.206.60/share/files/papers/virtual\%20humans/brief\%20virtual\%20reality \%20therapy\%20for $\% 20$ publi c\%20speaking\%20anxiety.pdf

Kahlon, S., Lindner, P., \& Nordgreen, T. (2019). Virtual reality exposure therapy for adolescents with fear of public speaking: A non-randomized feasibility and pilot study. Child and Adolescent Psychiatry and Mental Health, 13(1). doi:10.1186/s13034-019-0307-y

Lindner, P., Dagöö, J., Hamilton, W., Miloff, A., Andersson, G., Schill, A., \& Carlbring, P. (2020). Virtual Reality exposure therapy for public speaking anxiety in routine care: A single-subject effectiveness trial. Cognitive Behaviour Therapy, 1-21. doi:10.1080/16506073.2020.1795240

Lindner, P., Miloff, A., Zetterlund, E., Reuterskiöld, L., Andersson, G., \& Carlbring, P. (2019). Attitudes Toward and Familiarity With Virtual Reality Therapy Among Practicing Cognitive Behavior Therapists: A Cross-Sectional Survey Study in the Era of Consumer VR Platforms. Frontiers in Psychology, 10.

https://doi.org/10.3389/fpsyg.2019.00176 
Pan, X., \& Hamilton, A. F. D. C. (2018). Why and how to use virtual reality to study human social interaction: The challenges of exploring a new research landscape. British Journal of Psychology, 109(3), 395-417.

https://doi.org/10.1111/bjop.12290

Scruton, R. (2010). Hiding Behind the Screen. The New Atlantis, (28), 48-60. Retrieved October 28, 2020, from http://www.jstor.org/stable/43152971

Slater, M., Pertaub, D., \& Steed, A. (1999). Public speaking in virtual reality: Facing an audience of avatars. IEEE Computer Graphics and Applications, 19(2), 6-9. doi:10.1109/38.749116

Stupar-Rutenfrans, S., Ketelaars, L. E., \& Gisbergen, M. S. (2017). Beat the Fear of Public Speaking: Mobile 360 Video Virtual Reality Exposure Training in Home Environment Reduces Public Speaking Anxiety.

Cyberpsychology, Behavior, and Social Networking, 20(10), 624-633. doi:10.1089/cyber.2017.0174

Vera, L., Herrera, G., \& Vived, E. (2005). Virtual reality school for children with learning difficulties. Proceedings of the 2005 ACM SIGCHI International Conference on Advances in Computer Entertainment Technology - ACE '05. https://doi.org/10.1145/1178477.1178541 